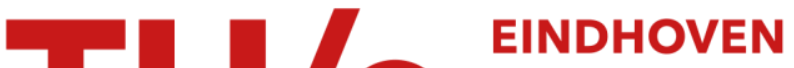 UNIVERSITY OF TECHNOLOGY
}

\section{Stabilization of networked control systems via non-monotone control Lyapunov functions}

\section{Citation for published version (APA):}

Gielen, R. H., \& Lazar, M. (2009). Stabilization of networked control systems via non-monotone control Lyapunov functions. In Proceedings Joint 48th IEEE Conference on Decision and Control and 28th Chinese Control Conference, 16-18 December 2009, Shanghai, China (pp. 7942-7948). Institute of Electrical and Electronics Engineers.

\section{Document status and date:}

Published: 01/01/2009

\section{Document Version:}

Publisher's PDF, also known as Version of Record (includes final page, issue and volume numbers)

\section{Please check the document version of this publication:}

- A submitted manuscript is the version of the article upon submission and before peer-review. There can be important differences between the submitted version and the official published version of record. People interested in the research are advised to contact the author for the final version of the publication, or visit the $\mathrm{DOI}$ to the publisher's website.

- The final author version and the galley proof are versions of the publication after peer review.

- The final published version features the final layout of the paper including the volume, issue and page numbers.

Link to publication

\section{General rights}

Copyright and moral rights for the publications made accessible in the public portal are retained by the authors and/or other copyright owners and it is a condition of accessing publications that users recognise and abide by the legal requirements associated with these rights.

- Users may download and print one copy of any publication from the public portal for the purpose of private study or research.

- You may not further distribute the material or use it for any profit-making activity or commercial gain

- You may freely distribute the URL identifying the publication in the public portal.

If the publication is distributed under the terms of Article 25fa of the Dutch Copyright Act, indicated by the "Taverne" license above, please follow below link for the End User Agreement:

www.tue.nl/taverne

Take down policy

If you believe that this document breaches copyright please contact us at:

openaccess@tue.nl

providing details and we will investigate your claim. 


\title{
Stabilization of Networked Control Systems via Non-monotone Control Lyapunov Functions
}

\author{
Rob Gielen, Student Member IEEE and Mircea Lazar, Member IEEE
}

\begin{abstract}
This paper deals with stabilization of networked control systems (NCS) affected by uncertain time-varying delays and data packet dropouts. We point out that such network effects are likely to render the classical control Lyapunov function (CLF) method unfeasible, mainly due to the monotonic decreasing condition. To solve this problem we make use of a discrete-time equivalent of a control Lyapunov-Razumikhin function (CLRF), which is allowed to be non-monotone. The corresponding stabilizing control law is obtained by solving an optimization problem on-line, in a receding horizon manner, which incorporates the available knowledge about the past state/input trajectory. Furthermore, we provide extra flexibility to the CLRF via a relaxation variable, which is needed to handle hard state/input constraints. We also propose an effective method for dealing with delays larger than the sampling period by means of an on-line Minkowski set addition. This makes it possible to guarantee stability even in the presence of data packet dropouts, under certain assumptions.
\end{abstract}

\section{INTRODUCTION}

Surveys on future directions in control, e.g. [1], identify networked control systems (NCS) to be one of the emerging key topics in control. The distinguishing feature of NCS is that the connection between plant and controller is made through a communication network. This brings specific, additional challenges to controller design, as shown in the comprehensive NCS overviews [2], [3]. Typically two main issues can be identified: uncertain time-varying delays and data packet dropout.

Our focus lies on the problem of stabilizing controller synthesis in the presence of uncertain time-varying delays, data packet dropouts and state/input constraints. Recently, an increasingly popular solution to the problem of uncertain time-varying delays was obtained by over-approximating the delay-induced nonlinearity with a polytope [4], [5], [6], [7]. Thus, the discrete-time uncertain nonlinear model arising from a continuous-time linear system with time-varying delays can be transformed into an uncertain linear model affected by an additive term due to the previous input. By augmenting the state vector with the previous input [8] this further yields a higher dimension linear model with polytopic uncertainty. In this way robust control methods based on Lyapunov theory for uncertain linear systems become available. The control Lyapunov function (CLF) for the augmented state system provides a Lyapunov-Krasovskii functional [9] for the non-augmented system, which establishes stability in the presence of time-varying delays.

R.H. Gielen and M. Lazar are with the Department of Electrical Engineering, Eindhoven University of Technology, The Netherlands, E-mails: r.h.gielen@tue.nl, m.lazar@tue.nl.
However, this approach has certain limitations. Firstly, imposing the stability conditions for all possible values of the augmented state vector may be conservative. Secondly, these LMI based off-line synthesis methods, e.g. [3], [5], yield a static feedback controller that can be conservative in the presence of state/input constraints. As such, it would be desirable to construct a stabilization method that is directly applicable to the non-augmented system, takes into account the available knowledge of the past state/input trajectory and can handle hard constraints. Unfortunately, a classical CLF is likely to be unfeasible when applied to the non-augmented system, as the past input(s) act(s) on the system state as an uncertain additive disturbance that can cause violation of the monotonic decrease condition.

In this paper we propose a novel stabilization method for NCS based on a control Lyapunov-Razumikhin function (CLRF). A Lyapunov-Razumikhin function (LRF) (originally defined for continuous-time systems [10]) is a function that is required to decrease only if a certain condition on the past and current states is satisfied. This condition allows for a non-monotonic behavior. However, the exact translation of this approach for discrete-time systems yields a noncausal constraint [11]. A feasible solution for discrete-time systems was devised in [12], where the LRF is required to be less than the maximum over its past values within a time window of length equal to the size of the delay. Inspired by this solution, we define a local CLRF candidate for the uncertain non-augmented linear model affected by an additive term due to the previous input [7]. Notice that using a CLRF already relaxes the monotonicity property of a classical CLF. Then, we construct an optimization problem (to be solved on-line) that enforces stability by an explicit CLRF-type constraint and includes state/input constraints as well. To handle the latter constraints, we provide a solution for further relaxing the CLRF-type condition by introducing an auxiliary optimization variable that adds extra flexibility to the local CLRF. Convergence to the equilibrium is still guaranteed via a particular limiting condition on the auxiliary optimization variable. Next, we show that delays larger than the sampling period can be handled efficiently by means of on-line Minkowski set additions. This is a notable feature of the proposed method, as existing analysis and synthesis methods, e.g. [5], based on uncertain linear models suffer from an exponential increase in complexity when delays larger than the sampling period are considered. Dealing efficiently with large delays is desirable also because then stability can be guaranteed even in the presence of packet dropouts, as shown in this paper. Using an infinity-norm 
based CLRF allows us to implement the proposed method as a single linear program. The developed theory is validated on a benchmark automotive case study: control of an active suspension system over a network.

\section{PRELIMINARIES}

\section{A. Notation}

Let $\mathbb{R}, \mathbb{R}_{+}, \mathbb{Z}$ and $\mathbb{Z}_{+}$denote the field of real numbers, the set of non-negative reals, the set of integers and the set of non-negative integers respectively. We use the notation $\mathbb{Z}_{\geq c_{1}}$ and $\mathbb{Z}_{\left(c_{1}, c_{2}\right]}$ to denote the sets $\left\{k \in \mathbb{Z}_{+} \mid k \geq c_{1}\right\}$ and $\left\{k \in \mathbb{Z}_{+} \mid c_{1}<k \leq c_{2}\right\}$, respectively, for some $c_{1}, c_{2} \in \mathbb{Z}_{+}$. For a vector $x \in \mathbb{R}^{n}$ let $\|$. $\|$ denote an arbitrary $p$ norm and let $[x]_{i}, i \in \mathbb{Z}_{[1, n]}$ denote the $i$-th component of $x$. Let $\|x\|_{\infty}:=\max _{i \in \mathbb{Z}_{[1, n]}}\left|[x]_{i}\right|$, where $|\cdot|$ denotes the absolute value. For a matrix $Z \in \mathbb{R}^{m \times n}$ let $Z^{-L}:=$ $\left(Z^{T} Z\right)^{-1} Z^{T}$ denote the left Moore-Penrose pseudoinverse. Let $\mathbf{z}:=\left\{z_{l}\right\}_{l \in \mathbb{Z}_{+}}$with $z_{l} \in \mathbb{R}^{n}$ for all $l \in \mathbb{Z}_{+}$denote an arbitrary sequence. Define $\|\mathbf{z}\|:=\sup \left\{\left\|z_{l}\right\| \mid l \in \mathbb{Z}_{+}\right\}$ and $\mathbf{z}_{[0, k]}:=\left\{z_{l}\right\}_{l \in \mathbb{Z}_{[0, k]}}$. A polyhedron, or a polyhedral set, in $\mathbb{R}^{n}$ is a set obtained as the intersection of a finite number of open and/or closed half-spaces. A polytope is a compact (closed and bounded) polyhedron. Given two polytopes $\mathcal{S}, \mathcal{P} \subseteq \mathbb{R}^{n}$ let $f_{0}(\mathcal{S})$ denote the number of vertices spanning $\mathcal{S}$ and let $\mathcal{S} \oplus \mathcal{P}:=\{x+y \mid x \in \mathcal{S}, y \in \mathcal{P}\}$ denote the Minkowski addition of $\mathcal{S}$ and $\mathcal{P}$. Let $\mathrm{Co}(\cdot)$ denote the convex hull and let $\operatorname{int}(\mathcal{S})$ denote the interior of an arbitrary set $\mathcal{S}$. A function $\varphi: \mathbb{R}_{+} \rightarrow \mathbb{R}_{+}$belongs to class $\mathcal{K}$ if it is continuous, strictly increasing and $\varphi(0)=0$. A function $\varphi: \mathbb{R}_{+} \rightarrow \mathbb{R}_{+}$is said to belong to class $\mathcal{K}_{\infty}$ if it is of class $\mathcal{K}$ and $\lim _{s \rightarrow \infty} \varphi(s)=\infty$.

\section{B. Stability of difference inclusions with time-delay}

Consider the discrete-time autonomous system

$$
x_{k+1} \in \Phi\left(\mathbf{x}_{[k-h, k]}\right), \quad k \in \mathbb{Z}_{+},
$$

where $\mathbf{x}_{[k-h, k]} \in \mathbb{X}^{h+1}:=\mathbb{X} \times \ldots \times \mathbb{X} \subseteq \mathbb{R}^{n} \times \ldots \times \mathbb{R}^{n}$, is the state trajectory starting with discrete-time instant $k-$ $h$ up to and including $k$, where $h \in \mathbb{Z}_{+}$is the maximal delay affecting the system and the mapping $\Phi: \mathbb{R}^{n} \times \ldots \times$ $\mathbb{R}^{n} \rightrightarrows \mathbb{R}^{n}$ is an arbitrary nonlinear set-valued function. We assume that system (1) has the origin as equilibrium point, i.e. $0 \in \operatorname{int}(\mathbb{X})$ and $\Phi\left(\mathbf{0}_{[-h, 0]}\right)=\{0\}$, where $\mathbf{0}_{[-h, 0]}$ denotes a sequence of zero vectors of appropriate dimension.

Definition II.1 (i) We call the origin of (1) attractive if for all $\mathbf{x}_{[-h, 0]} \in \mathbb{X}^{h+1}$ it holds that all corresponding state trajectories of (1) satisfy $\lim _{k \rightarrow \infty}\left\|x_{k}\right\|=0$; (ii) the origin of (1) is called Lyapunov stable (LS) if for every $\varepsilon>0$ there exists a $\delta(\varepsilon)>0$ such that, if $\left\|\mathbf{x}_{[-h, 0]}\right\|<\delta$ then all corresponding state trajectories of (1) satisfy $\left\|x_{k}\right\|<\varepsilon$ for all $k \in \mathbb{Z}_{+}$; (iii) We call system (1) asymptotically stable $(A S)$ if its origin is both attractive and LS.

Definition II.2 We call a set $\mathbb{X} \subseteq \mathbb{R}^{n}$ positively invariant (PI) for system (1) if for all $\mathbf{x}_{[-h, 0]} \in \mathbb{X}^{h+1}$ it holds that $\Phi\left(\mathbf{x}_{[-h, 0]}\right) \subseteq \mathbb{X}$.
Theorem II.3 Let $\mathbb{X}$ be a PI set for (1) with $0 \in \operatorname{int}(\mathbb{X})$. Let $\alpha_{1}, \alpha_{2} \in \mathcal{K}_{\infty}, \rho \in \mathbb{R}_{[0,1)}$. If there exists a function $V: \mathbb{R}^{n} \rightarrow$ $\mathbb{R}_{+}$such that

$$
\begin{array}{r}
\alpha_{1}(\|x\|) \leq V(x) \leq \alpha_{2}(\|x\|), \quad \forall x \in \mathbb{X}, \\
V\left(x^{+}\right) \leq \max _{i \in \mathbb{Z}_{[-h, 0]}} \rho V\left(x_{i}\right), \quad \forall \mathbf{x}_{[-h, 0]} \in \mathbb{X}^{h+1},
\end{array}
$$

for all $x^{+} \in \Phi\left(\mathbf{x}_{[-h, 0]}\right)$, then system (1) is AS.

The proof of the above theorem, which is omitted here for brevity, is similar in nature to the proof given in [12], Theorem 3.1, by replacing mutatis mutandis the difference equation with the difference inclusion as in (1). A function $V(\cdot)$ that satisfies the hypothesis of Theorem II.3 is called a Lyapunov-Razumikhin function (LRF). If $h=0$ then Theorem II.3 recovers the classical definition of a Lyapunov function (LF). Notice that a LRF is more general than a standard LF, as the maximum in (2b) allows the LRF to be locally non-monotone. It is worth to point out that the averaging condition applied recently in [13] to obtain a nonmonotone LF for discrete-time systems (without time-delay) is conceptually related to the Razumikhin condition.

Next, consider the non-autonomous system

$$
x_{k+1} \in \tilde{\phi}\left(\mathbf{x}_{[k-h, k]}, u_{k}\right), \quad k \in \mathbb{Z}_{+},
$$

where $\mathbf{x}_{[k-h, k]} \in \mathbb{X}^{h+1}, h \in \mathbb{Z}_{+}$is the maximum delay and $u_{k} \in \underset{\sim}{\mathbb{U}} \subseteq \mathbb{R}^{m}$ is the control input at the discrete-time instant $k . \tilde{\phi}: \mathbb{R}^{n} \times \ldots \times \mathbb{R}^{n} \times \mathbb{R}^{m} \rightrightarrows \mathbb{R}^{n}$ is an arbitrary setvalued function with $\tilde{\phi}\left(\mathbf{0}_{[-h, 0]}, 0\right)=\{0\}$. We assume that $0 \in \operatorname{int}(\mathbb{X})$ and $0 \in \operatorname{int}(\mathbb{U})$. Next, let $\alpha_{1}, \alpha_{2} \in \mathcal{K}_{\infty}$ and let $\rho \in \mathbb{R}_{[0,1)}$.

Definition II.4 A function $V: \mathbb{R}^{n} \rightarrow \mathbb{R}_{+}$that satisfies

$$
\alpha_{1}(\|x\|) \leq V(x) \leq \alpha_{2}(\|x\|), \quad \forall x \in \mathbb{R}^{n},
$$

and for which there exists a control law, possibly set-valued, $\pi: \mathbb{R}^{n} \times \ldots \times \mathbb{R}^{n} \rightrightarrows \mathbb{U}$ such that

$$
V\left(x^{+}\right) \leq \max _{i \in \mathbb{Z}_{[-h, 0]}} \rho V\left(x_{i}\right), \quad \forall \mathbf{x}_{[-h, 0]} \in \mathbb{X}^{h+1}
$$

for all $u \in \pi\left(\mathbf{x}_{[-h, 0]}\right)$ and all $x^{+} \in \tilde{\phi}\left(\mathbf{x}_{[-h, 0]}, u\right)$ is called a control Lyapunov-Razumikhin function (CLRF) for the difference inclusion corresponding to system (3) with $u_{k} \in \pi\left(\mathbf{x}_{[k-h, k]}\right)$ for all $k \in \mathbb{Z}_{+}$.

\section{NCS model for controller design}

Consider the continuous time system with input delay

$$
\begin{aligned}
& \dot{x}(t)=A_{c} x(t)+B_{c} u(t), \\
& u(t)=u_{k}, \quad \forall t \in\left[t_{k}+\tau_{k}, t_{k+1}+\tau_{k+1}\right),
\end{aligned}
$$

where $t_{k}=k T_{s}, k \in \mathbb{Z}_{+}, T_{s} \in \mathbb{R}_{+}$denotes the sampling period and we assume that $u(t)=u_{\text {initial }}$ for all $t \in\left[0, \tau_{0}\right)$ with $u_{\text {initial }} \in \mathbb{R}^{m}$ some predetermined constant vector. $A_{c} \in$ $\mathbb{R}^{n \times n}, B_{c} \in \mathbb{R}^{n \times m}$ are the system matrices, $u_{k} \in \mathbb{R}^{m}$ is the control action generated at time $t=t_{k}, u(t) \in \mathbb{R}^{m}$ is the system input and $x(t) \in \mathbb{R}^{n}$ is the system state. $\tau_{k} \in \mathbb{R}_{[0, \bar{\tau})}$ denotes the delay induced by the network at time $k \in \mathbb{Z}_{+}$ 
and $\bar{\tau} \in \mathbb{R}_{+}$is the maximal possible delay. For simplicity, we assume that $\bar{\tau} \in \mathbb{R}_{\left[0, T_{s}\right]}$, i.e. the maximal delay is smaller than or equal to the sampling period. The case of delays larger than the sampling period will be treated in Section IV.

Following the steps presented in [7] we obtain a discretetime model of (6), i.e.

$$
x_{k+1}=A_{d} x_{k}+B_{d} u_{k}+\Delta\left(\tau_{k}\right)\left(u_{k-1}-u_{k}\right), \quad k \in \mathbb{Z}_{+},
$$

where $A_{d}=e^{A_{c} T_{s}}, B_{d}=\int_{0}^{T_{s}} e^{A_{c}\left(T_{s}-\theta\right)} d \theta B_{c}$ and $\Delta\left(\tau_{k}\right)=$ $\int_{0}^{\tau_{k}} e^{A_{c}\left(T_{s}-\theta\right)} d \theta B_{c}$. The remaining challenge is to find a polytopic over-approximation of the nonlinear function $\Delta\left(\tau_{k}\right)$. Therefore, we define the following polytopic set

$$
\Delta^{\bar{\tau}}:=\operatorname{Co}\left(\left\{\bar{\Delta}_{l}\right\}_{l \in \mathbb{Z}_{[1, L]}}\right),
$$

with $\bar{\Delta}_{l} \in \mathbb{R}^{n \times m}$ such that $\Delta\left(\tau_{k}\right) \in \Delta^{\bar{\tau}}$ for all $\tau_{k} \in \mathbb{R}_{[0, \bar{\tau}]}$ and $L \in \mathbb{Z}_{\geq 1}$ is finite. In [7] several methods to create the polytope (8) were assessed. We refer to this reference for further details and assume for the remainder of this paper that the polytopic set (8) is known. Thus, (7) becomes

$$
x_{k+1} \in \phi\left(x_{k}, u_{k}, u_{k-1}\right), \quad k \in \mathbb{Z}_{+},
$$

where $u_{-1}$ is equal to some fixed, predetermined value and

$$
\begin{aligned}
& \phi\left(x_{k}, u_{k}, u_{k-1}\right):= \\
& \quad\left\{A_{d} x_{k}+B_{d} u_{k}+E_{\Delta}\left(u_{k-1}-u_{k}\right) \mid E_{\Delta} \in \Delta^{\bar{\tau}}\right\} .
\end{aligned}
$$

\section{Problem formulation}

Now the problem of interest is how to design a feedback control law that stabilizes system (9). This will guarantee stability of system (7) for all $\tau_{k} \in \mathbb{R}_{[0, \bar{\tau}]}$ because all possible realizations are taken into account via $E_{\Delta}$. Furthermore, even stability of the original continuous-time system (6) can be guaranteed under certain assumptions, as shown in [5], Chapter 4 (Section 4.3). As observed in the introduction classical CLFs are likely to be unfeasible for NCS affected by uncertain delays and subject to hard state/input constraints. Obviously, a non-monotone CLF would be more suitable, which motivates and justifies the novel stabilization method proposed in this paper.

\section{MAIn RESUlts}

For clarity of exposition, without loss of generality, in this section we assume that the delay is smaller than or equal to the sampling period. Consider the following inequality corresponding to (5)

$$
V\left(x^{+}\right) \leq \max _{i \in \mathbb{Z}_{[-1,0]}} \rho V\left(x_{k+i}\right)+\lambda_{k}, \quad \forall k \in \mathbb{Z}_{+},
$$

for all $x^{+} \in \phi\left(x_{k}, u_{k}, u_{k-1}\right)$. Here $\lambda_{k}$ is a variable which allows for additional freedom in the evolution of the CLRF, i.e. it can increase if (5) is too conservative at time instant $k \in \mathbb{Z}_{+}$, possibly due to active state/input constraints (see Section VI for an illustration). Based on (10) we formulate the following optimization problem.

Let $\alpha_{3}, \alpha_{4} \in \mathcal{K}_{\infty}$ and $J: \mathbb{R} \rightarrow \mathbb{R}_{+}$be an arbitrary function such that $\alpha_{3}(|\lambda|) \leq J(\lambda) \leq \alpha_{4}(|\lambda|)$ for all $\lambda \in \mathbb{R}$. Let $V(\cdot)$ be a candidate local CLRF for system (9).
Problem III.1 Assume that at time $k \in \mathbb{Z}_{+}, x_{k}, x_{k-1}$ and $u_{k-1}$ are known $^{1}$ and minimize the cost $J\left(\lambda_{k}\right)$ over $\lambda_{k}$ subject to

$$
\begin{aligned}
& u_{k} \in \mathbb{U}, \phi\left(x_{k}, u_{k}, u_{k-1}\right) \subseteq \mathbb{X}, \lambda_{k} \geq 0 \\
& V\left(x^{+}\right) \leq \max _{i \in \mathbb{Z}_{[-1,0]}} \rho V\left(x_{k+i}\right)+\lambda_{k},
\end{aligned}
$$

for all $x^{+} \in \phi\left(x_{k}, u_{k}, u_{k-1}\right)$.

Let $\pi\left(x_{k}, u_{k-1}\right):=\left\{u_{k} \in \mathbb{R}^{m} \mid \exists \lambda_{k} \in \mathbb{R}\right.$ s.t. (11) holds $\}$ and let $\phi_{\mathrm{cl}}\left(x_{k}, \pi\left(x_{k}, u_{k-1}\right), u_{k-1}\right):=\left\{\phi\left(x_{k}, u, u_{k-1}\right) \mid u \in\right.$ $\left.\pi\left(x_{k}, u_{k-1}\right)\right\}$. Furthermore, let $\mathcal{V}_{\Gamma}:=\left\{x \in \mathbb{R}^{n} \mid V(x) \leq \Gamma\right\}$ for any $\Gamma \in \mathbb{R}_{+}$. Let $\lambda_{k}^{*}$ denote the optimum in Problem III.1 for all $k \in \mathbb{Z}_{+}$.

Theorem III.2 Suppose that $V(\cdot)$ is a function that satisfies (4) and that $\mathbb{X}$ and $\mathbb{U}$ are bounded. Furthermore, suppose that Problem III.1 is feasible for all $\left(\mathbf{x}_{[-1,0]}, u_{-1}\right) \in \mathbb{X}^{2} \times \mathbb{U}$ and that $\lim _{k \rightarrow \infty} \lambda_{k}^{*}=0$. Then the origin of the difference inclusion

$$
x_{k+1} \in \phi_{c l}\left(x_{k}, \pi\left(x_{k}, u_{k-1}\right), u_{k-1}\right), \quad k \in \mathbb{Z}_{+},
$$

is attractive. Moreover, if $\exists \Gamma \in \mathbb{R}_{>0}$ such that $V(\cdot)$ is a CLRF for initial conditions in $\mathcal{V}_{\Gamma}$ for system (9), then system (12) is $A S$.

The next Lemma, which is proven in the Appendix, is needed to prove Theorem III.2.

Lemma III.3 Let $\rho \in \mathbb{R}_{[0,1)}$ and consider a sequence $\left\{\lambda_{i}\right\}_{i \in \mathbb{Z}_{+}}$with $\lambda_{i} \in \mathbb{R}_{+}$and bounded for all $i \in \mathbb{Z}_{+}$. Suppose that $\lim _{i \rightarrow \infty} \lambda_{i}=0$. Then $\lim _{k \rightarrow \infty} \sum_{i=0}^{k} \rho^{k-i} \lambda_{i}=0$.

Next, we prove Theorem III.2.

Proof: As Problem III.1 is feasible for all $\left(\mathbf{x}_{[-1,0]}, u_{-1}\right) \in \mathbb{X}^{2} \times \mathbb{U}$, it remains feasible for all $k \in \mathbb{Z}_{+}$ due to constraint (11a). As such, $\mathbb{X}$ is a PI set for system (12) and the inequality (11b) can be applied recursively. As

$$
\lambda_{k}^{*} \leq \sup _{\mathbf{x}_{[-1,0]} \in \mathbb{X}^{2}, y, z \in \mathbb{U}, x^{+} \in \phi\left(x_{0}, y, z\right)}\left(V\left(x^{+}\right)-\max _{i \in \mathbb{Z}_{[-1,0]}} \rho V\left(x_{i}\right)\right),
$$

for all $k \in \mathbb{Z}_{+}$, where the supremum exists due to boundedness of $\mathbb{X}, \mathbb{U}$ and $\Delta^{\bar{\tau}}$, continuity of $\phi(\cdot, \cdot, \cdot)$ in all arguments and continuity of the bounds (4) on $V(\cdot)$, we have that $\lambda_{k}^{*}$ is bounded for all $k \in \mathbb{Z}_{+}$. Next, let $\hat{\rho}=\rho^{1 / 2}$, let $i_{k}^{*}:=\arg \max _{i \in \mathbb{Z}_{[-1,0]}} \hat{\rho}^{-(k+i)} V\left(x_{k+i}\right)$ and let

$$
U_{k}:=\max _{i \in \mathbb{Z}_{[-1,0]}} \hat{\rho}^{-(k+i)} V\left(x_{k+i}\right) .
$$

We will prove that $U_{k+1} \leq U_{k}+\hat{\rho}^{-(k+1)} \lambda_{k}^{*}$ for all $k \in \mathbb{Z}_{+}$. If $i_{k+1}^{*}=0$ then for all $x_{k+1} \in \phi_{\mathrm{cl}}\left(x_{k}, \pi\left(x_{k}, u_{k-1}\right), u_{k-1}\right)$

\footnotetext{
${ }^{1}$ The state trajectory is obtained by choosing a particular input out of the feasible set defined by the constraints (11) at every time instant. A possible criterion for selecting the control input is presented in Section V. However, the stability result of Theorem III.2 is not influenced by the input choice.
} 
by (11b) it holds that

$$
\begin{aligned}
U_{k+1} & =\hat{\rho}^{-(k+1)} V\left(x_{k+1}\right) \\
& \leq \hat{\rho}^{-(k+1)}\left(\max _{i \in \mathbb{Z}_{[-1,0]}} \hat{\rho}^{2} V\left(x_{k+i}\right)+\lambda_{k}^{*}\right) \\
& \leq \max _{i \in \mathbb{Z}_{[-1,0]}} \hat{\rho}^{-(k+i)} V\left(x_{k+i}\right)+\hat{\rho}^{-(k+1)} \lambda_{k}^{*} \\
& =U_{k}+\hat{\rho}^{-(k+1)} \lambda_{k}^{*} .
\end{aligned}
$$

Furthermore, if $i_{k+1}^{*}=-1$ it holds that

$$
\begin{aligned}
U_{k+1} & =\hat{\rho}^{-(k+1-1)} V\left(x_{k+1-1}\right) \\
& \leq \max _{i \in \mathbb{Z}_{[-1,0]}} \hat{\rho}^{-(k+i)} V\left(x_{k+i}\right)=U_{k} .
\end{aligned}
$$

Therefore, as $U_{k} \leq U_{k}+\hat{\rho}^{-(k+1)} \lambda_{k}^{*}$ from (14) and (15) it follows that $U_{k+1} \leq U_{k}+\hat{\rho}^{-(k+1)} \lambda_{k}^{*}$ for all $k \in \mathbb{Z}_{+}$. Applying this inequality recursively yields

$$
\begin{aligned}
U_{k} & \leq U_{0}+\sum_{i=0}^{k-1} \hat{\rho}^{-(i+1)} \lambda_{i}^{*} \\
& \leq \max _{i \in \mathbb{Z}_{[-1,0]}} V\left(x_{i}\right)+\sum_{i=0}^{k-1} \hat{\rho}^{-(i+1)} \lambda_{i}^{*}, \quad \forall k \in \mathbb{Z}_{+} .
\end{aligned}
$$

By (13) and (16) we obtain

$$
V\left(x_{k}\right) \leq \hat{\rho}^{k} U_{k} \leq \hat{\rho}^{k} \max _{i \in \mathbb{Z}_{[-1,0]}} V\left(x_{i}\right)+\hat{\rho}^{-1} \sum_{i=0}^{k-1} \hat{\rho}^{k-i} \lambda_{i}^{*},
$$

for all $k \in \mathbb{Z}_{+}$. Next, from (4), (17) and the inequality $\alpha_{1}^{-1}(y+z) \leq \alpha_{1}^{-1}(2 \max \{y, z\}) \leq \alpha_{1}^{-1}(2 y)+\alpha_{1}^{-1}(2 z)$ it follows that

$$
\left\|x_{k}\right\| \leq \alpha_{1}^{-1}\left(2 \hat{\rho}^{k} \max _{i \in \mathbb{Z}_{[-1,0]}} \alpha_{2}\left(x_{i}\right)\right)+\alpha_{1}^{-1}\left(2 \hat{\rho}^{-1} \sum_{i=0}^{k-1} \hat{\rho}^{k-i} \lambda_{i}^{*}\right),
$$

where $\alpha_{1}^{-1} \in \mathcal{K}_{\infty}$. As $\hat{\rho} \in \mathbb{R}_{[0,1)}$ this yields

$$
\begin{aligned}
\lim _{k \rightarrow \infty}\left\|x_{k}\right\| \leq & \lim _{k \rightarrow \infty} \alpha_{1}^{-1}\left(2 \hat{\rho}^{k} \max _{i \in \mathbb{Z}_{[-1,0]}} \alpha_{2}\left(x_{i}\right)\right) \\
& +\lim _{k \rightarrow \infty} \alpha_{1}^{-1}\left(2 \hat{\rho}^{-1} \sum_{i=0}^{k-1} \hat{\rho}^{k-i} \lambda_{i}^{*}\right)=0,
\end{aligned}
$$

where the second term in the above sum vanishes by Lemma III.3. Observing that (18) holds for all $x_{k+1} \in$ $\phi_{\mathrm{cl}}\left(x_{k}, \pi\left(x_{k}, u_{k-1}\right), u_{k-1}\right)$ and all $k \in \mathbb{Z}_{+}$establishes attractivity.

Attractivity of the origin for system (12) further implies that all state trajectories $x_{k}$ reach the set $\mathcal{V}_{\Gamma}$ in finite time. Hence, there exists a $j\left(x_{0}\right) \in \mathbb{Z}_{+}$such that $V(\cdot)$ is a (local) CLRF for the system (12) for all $k \in \mathbb{Z}_{\geq j\left(x_{0}\right)}$. Moreover, in $\mathcal{V}_{\Gamma}$ there exists a corresponding admissible feedback law $u_{k}=\pi_{\Gamma}\left(\mathbf{x}_{[k-h, k]}\right), k \in \mathbb{Z}_{+}$. From Theorem II.3 it then follows that the origin of (12) is LS, which completes the proof.

Notice that the above proof applies mutatis mutandis to the general case when $i \in \mathbb{Z}_{[-h, 0]}$ for any $h \in \mathbb{Z}_{+}$, i.e. it is not limited to delays smaller than the sampling period. Next, we provide a non-conservative solution for guaranteeing that $\lim _{k \rightarrow \infty} \lambda_{k}^{*}=0$.

Lemma III.4 Let $N \in \mathbb{Z}_{\geq 1}$ be a fixed constant to be chosen a priori and let $\rho \in \mathbb{R}_{[0,1)}$. If

$$
0 \leq \lambda_{k} \leq \max _{i \in[1, N]} \rho^{i} \lambda_{k-i}^{*}, \quad \forall k \in \mathbb{Z}_{\geq N}
$$

then $\lim _{k \rightarrow \infty} \lambda_{k}=0$.

Proof: Since $\lambda_{k}^{*}$ is bounded for all $k \in \mathbb{Z}_{+}$, there exists an $M \in \mathbb{R}_{+}$such that $\lambda_{k}^{*} \leq M$ for $k \in \mathbb{Z}_{+}$. This and (19) implies

$$
\lambda_{k} \leq \rho^{k-N} \max _{i \in[1, N]} \rho^{i} \lambda_{N-i}^{*} \leq \rho^{k-N} \max _{i \in[1, N]} \rho^{i} M,
$$

for all $k \in \mathbb{Z}_{\geq N}$, where the first inequality can be proven via induction. Observing that $\max _{i \in[1, N]} \rho^{i} M=\rho M$ and $\rho \in \mathbb{R}_{[0,1)}$ concludes the proof.

By augmenting Problem III.1 with constraint (19) the property $\lim _{k \rightarrow \infty} \lambda_{k}^{*}=0$ is thus guaranteed, which is sufficient for attractivity. Notice that constraint (19) is not active for the first $N$ discrete-time instants, and then $\lambda_{k}$ can take any value in $\mathbb{R}_{[0, M]}$. Then, starting with $k=N$ it provides a monotonically decreasing upper bound $\bar{M}_{k}$ which allows $\lambda_{k}$ to take any value in $\mathbb{R}_{\left[0, \bar{M}_{k}\right]}$ for all $k \in$ $\mathbb{Z}_{\geq N}$. Obviously, if $\bar{M}_{k}=0$ for some $k$, which happens if $\lambda_{j}^{*}=0$ for all $j \in \mathbb{Z}_{[k-N+1, k]}$, constraint (19) is equivalent to $\lambda_{k}=0$, which is always feasible within $\mathcal{V}_{\Gamma}$. However, in real-life applications, where noise is present, or in case of reference tracking, the constraint (19) can become unfeasible. To maintain feasibility it suffices to re-initialize the algorithm, i.e. to discard (19) for the next $N$ discrete-time instants, as it is done initially.

\section{Large Delays and Packet Dropouts}

Consider, without loss of generality, the maximum delay $\bar{\tau}_{\text {large }}=(\Upsilon+v) T_{s}$, where $\Upsilon \in \mathbb{Z}_{\geq 1}$ and $v \in \mathbb{R}_{[0,1)}$. From here on we assume that if a control input that was generated previously to the active input arrives, then this older input is ignored. Assuming that $u_{k}=\psi_{k}$ for all $k \in \mathbb{Z}_{[-\Upsilon-1,-1]}$ with $\boldsymbol{\psi}_{[-\Upsilon-1,-1]}$ some predetermined vector and assuming that $\tau_{k} \in \mathbb{R}_{\left[0, \bar{\tau}_{\text {arge }}\right]}$ for all $k \in \mathbb{Z}_{+}$, (7) becomes

$$
\begin{aligned}
& x_{k+1}=A_{d} x_{k}+B_{d} u_{k}+\Delta_{0}\left(\tau_{k}\right)\left(u_{k-1}-u_{k}\right)+\Delta_{1}\left(\tau_{k}\right) \times \\
& \quad \times\left(u_{k-2}-u_{k-1}\right)+\ldots+\Delta_{\Upsilon}\left(\tau_{k}\right)\left(u_{k-\Upsilon-1}-u_{k-\Upsilon}\right),
\end{aligned}
$$

with

$\Delta_{i}\left(\tau_{k}\right):=\left\{\begin{array}{lc}0 & \tau_{k-i}-i T_{s} \leq 0 \\ \int_{0}^{\tau_{k-i}-i T_{s}} e^{A_{c}\left(T_{s}-\theta\right)} d \theta B_{c} & 0<\tau_{k-i}-i T_{s}<T_{s} \\ \int_{0}^{T_{s}} e^{A_{c}\left(T_{s}-\theta\right)} d \theta B_{c} & T_{s} \leq \tau_{k-i}-i T_{s}\end{array}\right.$

and $i \in \mathbb{Z}_{[0, \Upsilon]}$. Furthermore, letting $j \in \mathbb{Z}_{[0, \Upsilon]}$

$$
\Delta_{i}\left(\tau_{k}\right):=0 \quad \text { if } \quad \tau_{k-j} \leq \tau_{k-i}-(i-j) T_{s},
$$

for all $j<i$, i.e. a newer control update arrives before $u_{k-i}$ arrives and thus $u_{k-i}$ is ignored. 
Remark IV.1 Allowing for a maximal delay $\bar{\tau}_{\text {large }}=(1+$ $v) T_{s}$ is similar to allowing for a maximal delay $\bar{\tau}=v T_{s}$ and the possibility of one consecutive data packet dropout. When $\tau_{k}<\tau_{k-1}-T_{s}$, the input signal generated at time $k-1$ arrives after the signal generated at time $k$ and is therefore ignored. Thus, allowing for delays larger than the sampling period takes implicitly into account the possibility of a limited amount of data packet dropout.

Next, we show how the method proposed in this paper can be rendered computationally tractable for delays larger than the sampling period. For any $\chi \in \mathbb{R}_{\left[0, T_{s}\right]}$, let $\Delta^{\chi}$ be defined as $\Delta^{\bar{\tau}}$ in (8), such that $\Delta\left(\tau_{k}\right) \in \Delta^{\chi}$ for all $\tau_{k} \in \mathbb{R}_{[0, \chi]}$. As $\Delta_{i}\left(\tau_{k}\right) \in \Delta^{T_{s}}$ for all $i \in \mathbb{Z}_{[0, \Upsilon-1]}$ and $\Delta_{\Upsilon}\left(\tau_{k}\right) \in \Delta^{v T_{s}}$, we obtain that (20) is equivalent to

$$
x_{k+1} \in \hat{\phi}\left(x_{k}, u_{k}, u_{k-1}\right), \quad \forall k \in \mathbb{Z}_{+},
$$

where

$$
\begin{aligned}
& \hat{\phi}\left(x_{k}, u_{k}, u_{k-1}\right):=\left\{A_{d} x_{k}+B_{d} u_{k}\right. \\
& \left.\quad+E_{\Delta}\left(u_{k-1}-u_{k}\right)+v \mid E_{\Delta} \in \Delta^{T_{s}}, v \in \mathcal{S}_{k}\right\}
\end{aligned}
$$

and

$$
\begin{array}{r}
\mathcal{S}_{k}:=\left\{v \in \mathbb{R}^{n} \mid v\right. \\
\in \bigoplus_{i=1}^{\Upsilon-1} \Delta^{T_{s}}\left(u_{k-1-i}-u_{k-i}\right) \\
\left.\oplus \Delta^{v T_{s}}\left(u_{k-1-\Upsilon}-u_{k-\Upsilon}\right)\right\} .
\end{array}
$$

Observe that the input vectors $u_{k-i}$ are known at time $k \in \mathbb{Z}_{+}$for all $i \in \mathbb{Z}_{\geq 1}$. The complexity of Problem III.1, i.e. imposing the conditions (11), is in practice mainly determined by the complexity of the polytopic set $\mathcal{S}_{k}$. Computing $\mathcal{S}_{k}$ at every discrete-time instant can be performed efficiently via existing tools for calculating Minkowski additions [14]. Moreover, recent research [15] has led to much faster algorithms for performing Minkowski additions.

As the number of vertices spanning a polytope resulting from a Minkowski addition is likely to be much smaller than all possible combinations of vertices spanning the original polytopes, this can lead to a major reduction in complexity of Problem III.1. However, determining an exact upper bound on the number of vertices spanning a polytope resulting from a Minkowski addition is a non-trivial problem that has recently attracted much interest [16], [17]. Next, we show a result that illustrates the reduction in generators that can be achieved by a Minkowski addition.

Lemma IV.2 Consider the set $\mathcal{S}_{k}$ when $m=1$, i.e. the system has a single input. Then $f_{0}\left(\mathcal{S}_{k}\right) \leq f_{0}\left(\Delta^{T_{s}}\right)^{2} f_{0}\left(\Delta^{v T_{s}}\right)$ for all $k \in \mathbb{Z}_{+}$.

Proof: We use the fact that the Minkowski addition is associative, commutative and for polytopes satisfies [18] the property that $\lambda \mathcal{P} \oplus \mu \mathcal{P}=(\lambda+\mu) \mathcal{P}$ for all $\lambda, \mu \in \mathbb{R}$ and $\lambda \mu \geq 0$, to obtain

$$
\mathcal{S}_{k}=\Delta^{T_{s}} \sigma_{+} \oplus\left(-\Delta^{T_{s}}\right) \sigma_{-} \oplus \Delta^{v T_{s}}\left(u_{k-\Upsilon-1}-u_{k-\Upsilon}\right),
$$

where $\quad \sigma_{+}:=\sum_{i \in J_{+}}\left|u_{k-i-1}-u_{k-i}\right|, \quad \sigma_{-}:=$ $\sum_{i \in J_{-}}\left|u_{k-i-1}-u_{k-i}\right|, \int_{+}:=\left\{i \in \mathbb{Z}_{[1, \Upsilon]} \mid u_{k-i-1}-u_{k-i}>\right.$ $0\}$ and $\int_{-}:=\left\{i \in \mathbb{Z}_{[1, \Upsilon]} \mid u_{k-i-1}-u_{k-i}<0\right\}$. The trivial upper bound [17] $f_{0}\left(\mathcal{S}_{k}\right) \leq f_{0}\left(\Delta^{T_{s}}\right) f_{0}\left(\Delta^{T_{s}}\right) f_{0}\left(\Delta^{v T_{s}}\right)$ completes the proof.

For the more general case when $m \neq 1$ we refer the interested reader to [16], [17] and the references therein.

\section{IMPLEMENTATION ISSUES}

In this section we show how a local CLRF defined using infinity norms and corresponding control law can be obtained. Moreover, we briefly recall [19], [20] how the results derived above can be formulated as a single linear program (LP). For simplicity of exposition we consider the case where $\bar{\tau} \leq T_{s}$, i.e. system (9). However, the developed techniques apply straightforwardly to system (21).

Lemma V.1 If there exist a full column-rank $P \in \mathbb{R}^{p \times n}$, for some $p \in \mathbb{Z}_{\geq n}$, and $K \in \mathbb{R}^{m \times n}$ such that

$$
\left\|\left[\begin{array}{ll}
P\left(A_{d}+\left(B_{d}-\bar{\Delta}_{l}\right) K\right) & \left.P \bar{\Delta}_{l} K\right]
\end{array}\right]\left[\begin{array}{cc}
P & 0 \\
0 & P
\end{array}\right]^{-L}\right\|_{\infty}-\rho \leq 0,
$$

for all $l \in \mathbb{Z}_{[1, L]}$, then (9) in closed loop with the controller $u_{k}=K x_{k}$ is $A S$ and $V(x)=\|P x\|_{\infty}$ is a (local) CLRF for system (9).

The proof of Lemma V.1 is similar to the proof of [19], Theorem IV.2, and is therefore omitted. Matrices $P$ and $K$ can be obtained by solving a nonlinear optimization problem [19]. The largest sublevel set of $V(\cdot)$ contained in the admissible set corresponding to the feedback gain $K$ provides a set $\mathcal{V}_{\Gamma}$ that satisfies the hypothesis of Theorem III.2.

Substituting the candidate CLRF in constraint (11b) yields

$$
\begin{aligned}
\| P\left(A_{d} x_{k}+B_{d} u_{k}+\right. & \left.E_{\Delta}\left(u_{k-1}-u_{k}\right)\right) \|_{\infty} \\
& \leq \max _{i \in \mathbb{Z}_{[-1,0]}} \rho\left\|P x_{k-i}\right\|_{\infty}+\lambda_{k},
\end{aligned}
$$

where $x_{k}, x_{k-1}, P, u_{k-1}$ and $\rho \in \mathbb{R}_{[0,1)}$ are known and furthermore $E_{\Delta} \in \Delta^{\bar{\tau}}$. Via several simple steps [20] the inequality (24) can be formulated as a single LP that can be solved online. Similar steps reduce (19) to an additional constraint for the same LP. Moreover, a cost that penalizes the one-step ahead predicted state and the control input, i.e. $J\left(x_{k}, u_{k}, u_{k-1}, \lambda_{k}\right):=\left\|Q x^{+}\right\|_{\infty}+\left\|R u_{k}\right\|_{\infty}+\left\|G \lambda_{k}\right\|_{\infty}$, where $x^{+} \in \phi\left(x_{k}, u_{k}, u_{k-1}\right)$, can be added to select a control input out of the feasible set and to improve closed-loop performance in terms of settling time. Minimization of this cost function can again be translated into a set of additional constraints for the LP, as shown in [20].

\section{Automotive Application Example}

We test the results derived in the previous sections on an active suspension system [21], [22] controlled over a network. The system can be described using the following 
discrete-time state space model

$$
x_{k+1}=\left[\begin{array}{cccc}
0.809 & 0.009 & 0 & 0 \\
-36.93 & 0.80 & 0 & 0 \\
0.191 & -0.009 & 1 & 0.01 \\
0 & 0 & 0 & 1
\end{array}\right] x_{k}+\left[\begin{array}{c}
0.0005 \\
0.0935 \\
-0.005 \\
-0.01
\end{array}\right] u_{k}
$$

An initial condition is chosen corresponding to a large road disturbance $x_{0}=\left[\begin{array}{llll}-0.09 & 1.91 & -0.006 & 0\end{array}\right]^{T}$. The input signal is constrained, i.e. $u_{k} \in \mathbb{R}_{[-5,5]}$. The discrete time model was derived using a sampling period $T_{s}=0.025 \mathrm{~s}$ and we assume a maximum delay of $\bar{\tau}_{\text {large }}=0.0375 \mathrm{~s}$. As $\bar{\tau}_{\text {large }}$ is smaller than $2 T_{s}$, on-line computation of a Minkowski addition is not required to obtain $\mathcal{S}_{k}$. Furthermore, we used $Q=\operatorname{diag}(10,1,20,1), R=0$ and $G=1$ in the cost $J\left(x_{k}, u_{k}, u_{k-1}, \lambda_{k}\right)$. Using the method presented in Section V we were able to find a local CLRF for system (25) up to and including $\bar{\tau}=0.01 s$. However, for larger values of $\bar{\tau}$ this method did not lead to a feasible solution. To show the power of the receding horizon approach, we computed the following local CLF (i.e. obtained by letting $\bar{\Delta}_{l}=0$ in (23)) along with a static state feedback law for system (25) and $\rho=0.9$, i.e.

$$
\begin{aligned}
P & =\left[\begin{array}{cccc}
84.1261 & 0.5171 & -24.8263 & 5.3504 \\
-1.1848 & 1.1690 & 9.3424 & -2.0188 \\
-5.0178 & 0.0070 & 41.0863 & -13.9880 \\
42.9517 & 0.1415 & -54.9821 & 16.1326
\end{array}\right], \\
K & =\left[\begin{array}{llll}
114.5375 & -6.1746 & 68.3356 & -16.9713
\end{array}\right] .
\end{aligned}
$$

The function $V(x)=\|P x\|_{\infty}$ and $\rho=0.9$ were used in (11b) and $N$ was set equal to 10 in (19). Notice that for this choice of $V(\cdot)$ feasibility of the corresponding optimization problem implies only attractivity. If $V(\cdot)$ turns out to be a local CLRF as well, than AS is attained. Further research is focused on computation of local CLRFs defined using the infinity norm.

Simulations were performed using Matlab 7.3.0 and Simulink on a $2.66 \mathrm{GHz}$ Pentium 4 desktop PC. The worst case time needed for computation of the control input was smaller than $5 \mathrm{~ms}$, which is well below the sampling period $T_{s}$. Figure 1 shows the time histories for the states and input. The frequent packet dropout and time-varying delays can be observed in Figure 2, while Figure 3 presents the time history of $\lambda_{k}^{*}$ and of the function $V\left(x_{k}\right)$. It can be seen that despite the conservative choice of the weight $P$ in the function $V(\cdot)$, due to the Razumikhin condition, which allows a nonmonotonic behavior, and due to the additional flexibility provided by $\lambda_{k}^{*}$, the closed-loop trajectory converges to the origin with a good performance despite severe time delays and packet dropouts. Furthermore, the simulation illustrates the benefits of using an additional relaxation on top of the Razumikhin condition, as a non-zero value for $\lambda_{k}^{*}$ is needed to obtain a feasible solution at several time-instants. This demonstrates the effectiveness of the developed method.
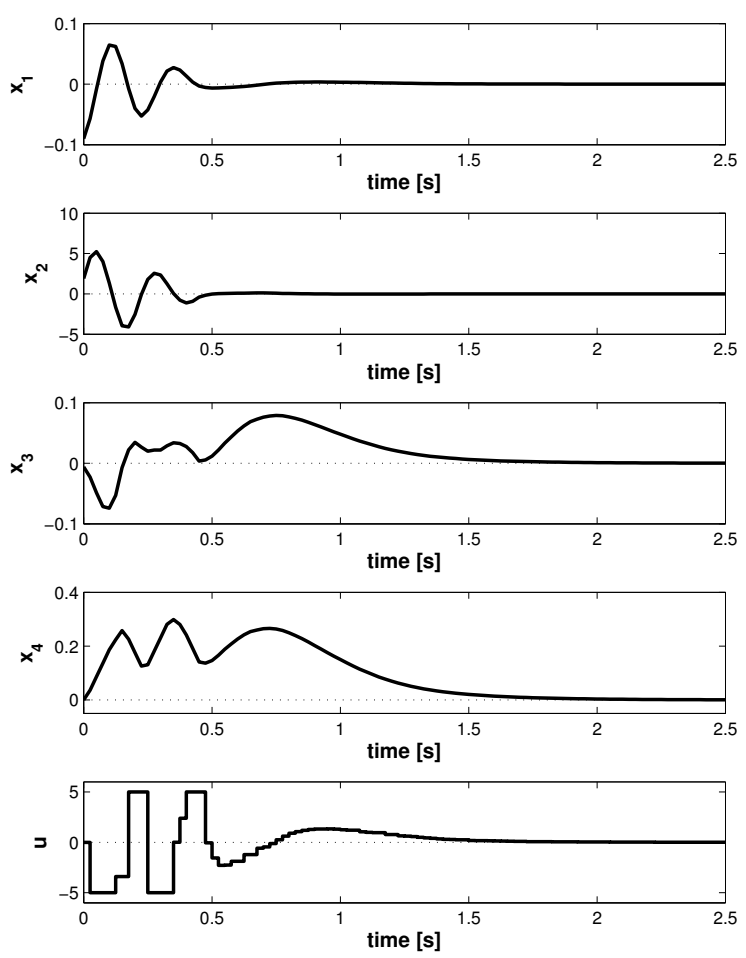

Fig. 1. Time history of the states and input.

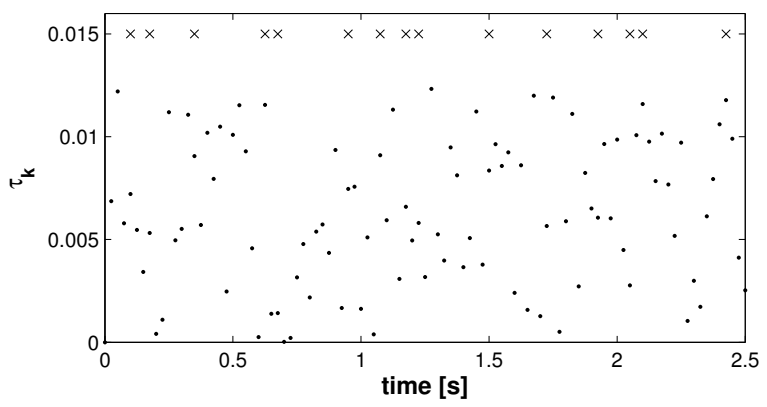

Fig. 2. Time history of the delay induced by the network $(\cdot)$ and the packet dropouts $(\mathrm{x})$.
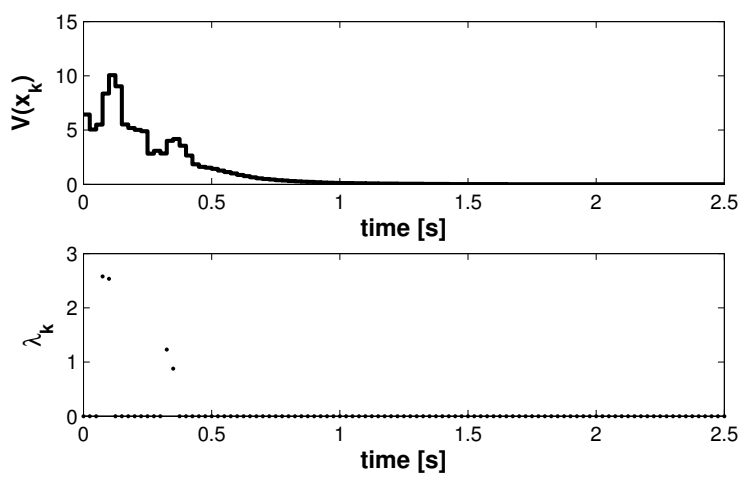

Fig. 3. Time history of $\lambda_{k}^{*}$ and $V\left(x_{k}\right)$. 


\section{CONCLUSIONS}

This paper was concerned with stabilization of networked control systems (NCS) affected by uncertain time-varying delays and data packet dropouts. We pointed out that such network effects are likely to render the classical control Lyapunov function (CLF) method unfeasible. To solve this problem, we have made use of a discrete-time equivalent of a control Lyapunov-Razumikhin function (CLRF), which is allowed to be non-monotone. Then, we have constructed an optimization problem to be solved on-line, in a receding horizon manner, which incorporates a CLRF-type condition as an explicit constraint. Furthermore, to deal with hard state/input constraints we have added extra flexibility to the CLRF via an auxiliary optimization variable that is minimized on-line under a limiting condition. We also proposed an effective method for dealing with delays larger than the sampling period by means of on-line Minkowski set additions. This made it possible to guarantee stability even in the presence of data packet dropouts, under certain assumptions.

\section{ACKNOWLEDGEMENTS}

The authors are grateful to Wim J.J. Gielen and Ilya V. Kolmanovsky for useful discussions. The research presented in this paper is supported by the Veni grant "Flexible Lyapunov Functions for Real-time Control", grant number 10230, awarded by STW and NWO.

\section{APPENDIX}

Proof of Lemma III.3: Firstly, recall the standard definition of a convergent sequence [23]. An arbitrary sequence $\left\{s_{k}\right\}_{k \in \mathbb{Z}_{+}}$of real numbers is convergent and has the limit $s$ if, $\forall \varepsilon$ there exists $N(\varepsilon) \in \mathbb{Z}_{+}$such that,

$$
\left|s_{k}-s\right|<\varepsilon, \quad \forall k \in \mathbb{Z}_{\geq N(\varepsilon)} .
$$

Also, observe that the boundedness of each $\lambda_{i} \geq 0$ implies the existence of an $M \in \mathbb{R}_{+}$such that $\lambda_{i} \leq M$ for all $i \in \mathbb{Z}_{+}$. Moreover, $\lim _{i \rightarrow \infty} \lambda_{i}=0$ implies that $\forall \varepsilon$ there exists an $i^{*}(\varepsilon) \in \mathbb{Z}_{+}$such that $\lambda_{i}<m:=\frac{\varepsilon}{2}(1-\rho)$ for all $i>i^{*}(\varepsilon)$. Consider now the sequence $s_{k}:=\sum_{i=0}^{k} \rho^{k-i} \lambda_{i}$ and the desired limit $s:=0$. Then, $\forall \varepsilon$ and for any $k>i^{*}(\varepsilon)$ it holds that

$$
\left|s_{k}-0\right|=\left|\sum_{i=0}^{k} \rho^{k-i} \lambda_{i}\right|<\left|\sum_{i=0}^{i^{*}(\varepsilon)} \rho^{k-i} M+\sum_{i=i^{*}(\varepsilon)+1}^{k} \rho^{k-i} m\right| .
$$

As $\left(\rho^{-1}-1\right) \sum_{i=0}^{i^{*}(\varepsilon)} \rho^{-i}=\rho^{-i^{*}(\varepsilon)-1}-1$, we further obtain

$$
\left|s_{k}-0\right|<\left|\frac{\rho^{k-i^{*}(\varepsilon)}-\rho^{k+1}}{1-\rho} M+\frac{1-\rho^{k-i^{*}(\varepsilon)}}{1-\rho} m\right| .
$$

Next, $\forall \varepsilon$ pick $N(\varepsilon)$ such that

$$
\frac{\rho^{N(\varepsilon)-i^{*}(\varepsilon)}-\rho^{N(\varepsilon)+1}}{1-\rho} M \leq \frac{\varepsilon}{2} .
$$

Then, from (28) it follows that (26) holds for all $k \in \mathbb{Z}_{\geq N(\varepsilon)}$ with $s=0$. This establishes the convergence of the series $s_{k}$ to the limit $s=0$, which completes the proof.

\section{REFERENCES}

[1] R. Murray, K. Åström, S. Boyd, R. Brockett, and G. Stein, "Future directions in control in an information-rich world," IEEE Control Systems Magazine, vol. 23, no. 2, pp. 20-33, 2003.

[2] Y. Tipsuwan and M.-Y. Chow, "Control methodologies in networked control systems," Control Engineering Practice, vol. 11, pp. 10991111,2003

[3] J. Hespanha, P. Naghshtabrizi, and Y. Xu, "A survey of recent results in networked control systems," in Proceedings of the IEEE, vol. 95, no. $1,2007$.

[4] L. Hetel, J. Daafouz, and C. Iung, "Stabilization of arbitrary switched linear systems with unknown time varying delays," IEEE Transactions on Automatic Control, vol. 51, no. 10, pp. 1668-1674, 2006.

[5] M. Cloosterman, "Control of systems over communication networks: Modelling, analysis and design," Ph.D. dissertation, Eindhoven University of Technology, The Netherlands, 2008.

[6] S. Olaru and S.-I. Niculescu, "Predictive control for linear systems with delayed input subject to constraints," in Proceedings of the 17th IFAC world congres (CD-ROM), Seoul, Korea, 2008.

[7] R. Gielen, S. Olaru, and M. Lazar, Nonlinear Model Predictive Control: Towards new challenging applications, ser. Lecture Notes in Control Sciences. Springer, 2009, vol. 384, ch. On Polytopic Approximations of Systems with Time-Varying Input Delays, pp. 225233.

[8] K. Åström and B. Wittenmark, Computer Controlled Systems, Theory and Design. Englewood Cliffs, NJ: Prentice Hall International, Inc., 1990.

[9] L. Hetel, J. Daafouz, and C. Iung, "Equivalence between the Lyapunov-Krasovskii functionals approach for discrete delay systems and that of the stability conditions for switched systems," Nonlinear Analysis: Hybrid Systems, vol. 51, no. 10, pp. 697-705, 2008.

[10] J. Hale, Theory of Functional Differential Equations. Springer-Verlag, New York, 1977.

[11] S. Elaydi and S. Zhang, "Stability and periodicity of difference equations with finite delay," Funkcialaj Ekvacioj, vol. 37, pp. 401413, 1994.

[12] B. Liu and H. Marquez, "Uniform stability of discrete delay systems and synchronization of discrete delay dynamical networks via Razumikhin technique," IEEE Transactions on Circuits and Systems, vol. 55, no. 9, pp. 2795-2805, 2008.

[13] A. A. Ahmadi and P. A. Parrilo, "Non-monotonic Lyapunov functions for stability of discrete time nonlinear and switched systems," in 47th IEEE Conference on Decision and Control, Cancun, Mexico, 2008, pp. 614-621.

[14] M. Kvasnica, P. Grieder, M. Baotic, and M. Morari, "Multi Parametric Toolbox (MPT)," in Hybrid Systems: Computation and Control, ser. Lecture Notes in Computer Science, Volume 2993. Pennsylvania, Philadelphia, USA: Springer-Verlag, mar 2004, pp. 448-462.

[15] H. Barki, F. Denis, and F. Dupont, "Contributing vertices-based Minkowski sum computation of convex polyhedra," Computer-Aided Design, vol. 41, pp. 525-538, 2009.

[16] R. Sanyal, "Toplogical obstructions for vertex numbers of Minkowski sums," Journal of Combinatorial Theory, Series A, vol. 116, pp. 168179, 2009.

[17] C. Weibel, "Minkowski sums of polytopes: Combinatorics and computation," Ph.D. dissertation, École Polytechnique Fédérale de Lausanne, Switzerland, 2007.

[18] B. Grünbaum, Convex Polytopes. Springer-Verlag, New York, 2003.

[19] M. Lazar, W. Heemels, S. Weiland, and A. Bemporad, "Stabilizing model predictive control of hybrid systems," IEEE Transactions on Automatic Control, vol. 51, no. 11, pp. 1813-1818, 2006.

[20] M. Lazar, "Flexible control Lyapunov functions," in American Control Conference, St. Louis, MO, 2009, pp. 102-107.

[21] D. Hrovat, "Survey of advanced suspension developments and related optimal control applications," Automatica, vol. 33, no. 10, pp. 1781$1817,1997$.

[22] A. Bemporad, F. Borrelli, and M. Morari, "Min-max control of constrained uncertain discrete-time linear systems," IEEE Transactions on Automatic Control, vol. 48, no. 9, pp. 1600-1606, 2003.

[23] J. Stewart, Calculus, Early Transcendentals. Thomson Learning Brooks/Cole, 2003. 